\title{
An Appraisal of Muon Neutrino Disappearance at Short Baseline
}

\author{
L. Stanco, ${ }^{1}$ S. Dusini, ${ }^{1}$ A. Longhin, ${ }^{2}$ A. Bertolin, ${ }^{1}$ and M. Laveder ${ }^{3}$ \\ ${ }^{1}$ INFN-Padova, Via Marzolo 8, 35131 Padova, Italy \\ ${ }^{2}$ Laboratori Nazionali di Frascati, INFN, Via E. Fermi 40, 00044 Frascati, Italy \\ ${ }^{3}$ Padova University and INFN-Padova, Via Marzolo 8, 35131 Padova, Italy \\ Correspondence should be addressed to L.Stanco; luca.stanco@pd.infn.it
}

Received 20 June 2013; Revised 12 September 2013; Accepted 6 October 2013

Academic Editor: Kate Scholberg

Copyright (C) 2013 L. Stanco et al. This is an open access article distributed under the Creative Commons Attribution License, which permits unrestricted use, distribution, and reproduction in any medium, provided the original work is properly cited.

\begin{abstract}
Neutrino physics is nowadays receiving more and more attention as a possible source of information for the long-standing problem of new physics beyond the Standard Model. The recent measurement of the third mixing angle $\theta_{13}$ in the standard mixing oscillation scenario encourages us to pursue the still missing results on leptonic CP violation and absolute neutrino masses. However, several puzzling measurements exist which deserve an exhaustive evaluation. We will illustrate the present status of the muon disappearance measurements at small $L / E$ and the current CERN project to revitalize the neutrino field in Europe with emphasis on the search for sterile neutrinos. We will then illustrate the achievements that a double muon spectrometer can make with regard to discovery of new neutrino states, using a newly developed analysis.
\end{abstract}

\section{Introduction}

The unfolding of the physics of the neutrino is a long and exciting story spanning the last 80 years. Over this time the interchange of theoretical hypotheses and experimental facts has been one of the most fruitful demonstrations of the progress of knowledge in physics. The work of the last decade and a half finally brought a coherent picture within the Standard Model (SM) (or some small extensions of it), namely, the mixing of three neutrino flavour states with three $v_{1}, v_{2}$, and $v_{3}$ mass eigenstates. The last unknown mixing angle, $\theta_{13}$, was recently measured [1-4], but still many questions remain unanswered to completely settle the scenario: the absolute masses, the Majorana/Dirac nature, and the existence and magnitude of leptonic CP violation. Answers to these questions will beautifully complete the (standard) three-neutrino model, but they will hardly provide an insight into new physics Beyond the Standard Model (BSM). Many relevant questions will stay open: the reason for neutrinos, the relation between the leptonic and hadronic sectors of the SM, the origin of Dark Matter, and overall where and how to look for BSM physics. Neutrinos may be an excellent source of BSM physics and their story is supporting that at length.
There are actually several experimental hints for deviations from the "coherent" picture described above. Many unexpected results, not statistically significant on a single basis, appeared also in the last decade and a half, bringing attention to the hypothesis of sterile neutrinos [5]. A recent White Paper [6] contains a comprehensive review of these issues. In this paper we will focus on one of the most intriguing and long-standing unresolved result: the unexpected oscillation of neutrinos at relatively small values of the ratio $L / E$ (distance in $\mathrm{km}$, energy in $\mathrm{GeV}$ ), corresponding to a scale of $\mathcal{O}(1) \mathrm{eV}^{2}$, incompatible with the much smaller values related to the atmospheric $\left|\Delta m_{32}^{2}\right| \simeq 2.4 \times 10^{-3} \mathrm{eV}^{2}$ and to the solar $\Delta m_{21}^{2} \simeq 8 \times 10^{-5} \mathrm{eV}^{2}$ scales.

The first unexpected measurement came from an excess of $\bar{\nu}_{e}$ originating from an initial $\bar{\nu}_{\mu}$ beam from Decay At Rest (LNSD [7]). The LSND experiment saw a $3.8 \sigma$ effect. The subsequent experiment with $\nu_{\mu}\left(\bar{v}_{\mu}\right)$ beam from accelerator, MiniBooNE [8-11], despite confirming an independent $3.8 \sigma$ effect after sustained experimental work, was unable to draw conclusive results on the origin of the LSND effect having observed an excess at higher $L / E$ in an energy region where background is high.

In recent years many phenomenological studies were performed by analyzing the LSND effect together with similar 
unexpected results coming from the measurement of lower than expected rates of $\bar{v}_{e}$ and $v_{e}$ interactions (disappearance), either from (a) near-by nuclear reactors $\left(\bar{\nu}_{e}\right)$ [12-14] or (b) from Mega-Curie $K$-capture calibration sources in the solar $v_{e}$ gallium experiments [15-21]. These $v_{e}\left(\bar{v}_{e}\right)$ disappearance measurements, all at the statistical level of 3-4 $\sigma$, could also be interpreted [22] as oscillations between neutrinos at large $\Delta m^{2} \simeq 1 \mathrm{eV}^{2}$. Several attempts were then tried to reach a coherent picture in terms of mixing between active and sterile neutrinos, in $3+1$ and $3+2[23,24]$ or even $3+1+1$ $[25,26]$ or $3+3$ [27] models, as extensions of the standard three-neutrino model. We refer to [28-30] as the most recent and industrious works where a very crucial issue is raised: " $a$ consistent interpretation of the global data in terms of neutrino oscillations is challenged by the non-observation of a positive signal in $v_{\mu}$ disappearance experiments" [29]. In fact, in any of the above models, essential information comes from the disappearance channels $\left(v_{\mu}\right.$ or $\left.v_{e}\right)$, which is one of the cleanest channels to measure the oscillation parameters (see Section 3 for details).

The presence of additional sterile states introduces quite naturally appearance and disappearance phenomena involving the flavour states in all channels. In particular, a $v_{\mu}$ disappearance effect has to be present and possibly measured. It turns out that only old experiments and measurements are available for Charged Current (CC) $\nu_{\mu}$ interactions at small $L / E$ [31]. The CDHS experiment reported in 1984 the nonobservation of $\nu_{\mu}$ oscillations in the $\Delta m^{2}$ range $0.3 \mathrm{eV}^{2}$ to $90 \mathrm{eV}^{2}$. Their analyzed region of oscillation did not span, however, low values of the mixing parameter down to around 0.1 in $\sin ^{2}(2 \theta)$. More recent results are available on $v_{\mu}$ disappearance from MiniBooNE [32], a joint MiniBooNE/SciBooNE analysis $[33,34]$ and the Long-Baseline MINOS experiment [35]. These results slightly extend the $\nu_{\mu}$ disappearance exclusion region, however, still leaving out the small-mixing region. Similar additional constraints on $v_{\mu}$ disappearance could possibly come from the analysis of atmospheric neutrinos in IceCube $[36,37]$.

Despite this set of measurements being rather unsatisfactory when compared with the corresponding LSND allowed region that lies at somewhat lower values of the mixing angle, they are still sufficient to introduce tensions in all the phenomenological models developed so far (see, e.g., $[6,28-30,38]$ for comprehensive and recent reviews). Therefore it is mandatory to set up a new experiment able to improve the small-mixing angle region exclusion by at least one order of magnitude with respect to the current results. In such a way one could also rule out the idea that the mixing angle extracted from LSND is larger than the true value due to a data overfluctuation. Once again, the $v_{\mu}$ disappearance channel should be the optimal one to perform a full disentangling of the mechanism given the strong tension between the $\nu_{e}$ appearance and $\nu_{\mu}$ disappearance around $\Delta m^{2} \simeq 1 \mathrm{eV}^{2}$. In fact, whereas the LSND effect may be confirmed by a more accurate $v_{e}$ oscillation measurement, only the presence of a $\nu_{\mu}$ oscillation pattern could shed more light on the nature and the interpretation of the effect.
Further, in the paper we will briefly discuss the newly proposed CERN experiment, following a detailed analysis of possible ways to measure the $v_{\mu}$ disappearance channel. In particular the evaluation of the $v_{\mu}$ disappearance rates at two different sites leads us naturally to take their ratio, elucidating the possibility to observe depletions and/or excesses. Throughout the paper we will focus on the need for a new $v_{\mu}$ CC measurement corresponding to an increase of one order of magnitude in the sensitivity to the mixing parameter, exploiting the determination of the muon charge for a proper evaluation of the different models and the separation of $\nu_{\mu}$ and $\bar{\nu}_{\mu}$ in the same neutrino beam. Finally, we will pay particular attention to the consistency and robustness (in statistical terms) of the results.

\section{The CERN Experimental Proposal}

The need for a definitive clarification on the possible existence of a neutrino mass scale around $1 \mathrm{eV}$ has brought up several proposals and experimental suggestions exploiting the sterile neutrino option by using different interaction channels and refurbished experiments. In the light of the considerations discussed in the previous section there are essentially two sets of experiments which must be redone: (a) the measurement of $\bar{v}_{e}$ neutrino fluxes at reactors (primarily the ILL one) together with refined and detailed computations of the flux simulations (see, e.g., [39, 40]); (b) the appearance/disappearance oscillation measurements at low $L / E$ with a standard muon neutrino beam with its intrinsic electron neutrino component.

There is actually another interesting option which comes from the Neutrino Factory studies and the very recently submitted EOI from $v$ STORM [41]. We refer to [42] for a comprehensive review of the corresponding possible $v_{e}$ and $v_{\mu}$ disappearance effects. It is interesting to note that our figures of merit about $v_{\mu}$ disappearance are rather similar to or even slightly more competitive than those illustrated in [42] (e.g., compare the exclusion regions in Figure 6 of [42] and those in Figure 7 of this paper despite the use of different C.L.), not forgetting the rather long time needed to set up the vSTORM project.

Coming to experiments with standard beams, investigations are underway at CERN where two physics proposals $[43,44]$ were submitted in October 2011 and later merged into a single technical proposal (ICARUS-NESSiE, [45]). CERN has subsequently set up working groups for the proton beam extraction from the SPS, the secondary beam line, and the needed infrastructure/buildings for the detectors. The work was reported in a recent LOI [46].

The experiment is based on two identical Liquid Argon (LAr)-Time Projection Chambers (TPC) [44] complemented by magnetized spectrometers [43] detecting electron and muon neutrino events at far and near positions, $1600 \mathrm{~m}$ and $460 \mathrm{~m}$ away from the proton target, respectively. The project will exploit the ICARUS T600 detector, the largest LAr-TPC ever built of about 600 ton mass, now presently in the LNGS underground laboratory where it was exposed to the CNGS beam. It is supposed to be moved at the CERN far 
position. An additional 1/4 of the T600 detector (T150) would be constructed from scratch as a clone of the original one, except for the dimensions, and located in the near site. Two spectrometers would be placed downstream of the two LArTPC detectors to greatly enhance the physics reach. The spectrometers will exploit a bipolar magnet with instrumented iron slabs, and a newly designed air-core magnet, to perform charge identification and muon momentum measurements in an extended energy range (from $0.5 \mathrm{GeV}$ or less to $10 \mathrm{GeV}$ ), over a transverse area larger than $50 \mathrm{~m}^{2}$.

While the LAr-TPCs will mainly perform a direct measurement of electron neutrinos [47], the spectrometers will allow an extended exploitation of the muon neutrino component, with neutrino/antineutrino discrimination on an eventby-event basis.

2.1. The Neutrino Beam. The proposed new neutrino beam will be constructed in the SPS North Area [46]. The setup is based on a $100 \mathrm{GeV}$ proton beam with a fast extraction scheme providing about $3.5 \cdot 10^{13}$ protons/pulse in two pulses of $10.5 \mu \mathrm{s}$ durations separated by $50 \mathrm{~ms}$ for a sample of about $4.5 \cdot 10^{19}$ protons on target (p.o.t.) per year (pulses of $10.5 \mu$ s duration are normally put in coincidence with the fast response of the spectrometers' detectors and efficiently used to reject the cosmic ray background. See, e.g., [48] where a time resolution of less than $2 \mathrm{~ns}$ is reported for the detectors used in the OPERA experiment). A target station will be located next to the so-called TCC 2 target zone, $11 \mathrm{~m}$ underground, followed by a cylindrical He-filled decay pipe with a length of about $110 \mathrm{~m}$ and a diameter of $3 \mathrm{~m}$. The beam dump of $15 \mathrm{~m}$ in length will be composed of iron blocks with a graphite inner core. Downstream of the beam dump a set of muon chambers stations will act as beam monitors. The beam will point upward, with a slope of about $5 \mathrm{mrad}$, resulting in a depth of $3 \mathrm{~m}$ for the detectors in the far site.

The current design of the focusing optics includes a pair of pulsed magnetic horns operated at relatively low currents. A graphite target of about $1 \mathrm{~m}$ in length is deeply inserted into the first horn allowing a large acceptance for the focusing of low momentum pions emitted at large angles. This design allows production of a spectrum peaking at about $2 \mathrm{GeV}$ thus matching the most interesting domain of $\Delta m^{2}$ with the detector locations at 460 and $1600 \mathrm{~m}$ from the target.

The charged current event rates for $\nu_{\mu}$ and $\bar{\nu}_{\mu}$ at the near and far detectors are shown in Figure 1 for the positive and negative focusing configuration.

A relevant contamination of $v_{\mu}$ in the negative polarity configuration is visible especially at high energy. This component arises as a result of the decays of high energy poorly defocused mesons produced at small angles. The charge discrimination of the magnetic system described below will allow an efficient discrimination of these two components with a charge confusion below or of the order of $1 \%$ from sub$\mathrm{GeV}(0.3-0.5 \mathrm{GeV})$ up to momenta around 8-10 GeV [43].

2.2. Spectrometer Requirements. The main purpose of the spectrometers placed downstream of the LAr-TPC is to provide charge identification and momentum reconstruction for the muons produced in neutrino interactions occurring in the LAr volume or in the magnetized iron of the spectrometers. In order to perform this measurement with high precision and in a wide energy range, from sub- $\mathrm{GeV}$ to multi$\mathrm{GeV}$, a massive iron-core dipole magnet (ICM) is coupled to an air-core magnet (ACM) in front of it [43]. Low momentum muons will be measured by the ACM, while the ICM will be employed at higher momenta.

As considered in the previous sections the definition of two sites, near and far, constitutes a fundamental issue for each physics program which aims to perform any sterile neutrino search. The two layouts have to be as similar as possible in order to allow an almost complete cancellation of the systematic uncertainties when comparing the measurements made at the near and far sites. Hence the near spectrometer will be an exact clone of the far one, with identical thickness along the beam axis but a scaled transverse size. A sketch of the possible far site NESSiE detector is shown in Figure 2.

The key feature of the ACM is the large geometric and momentum acceptance. The need of a low momentum threshold for muon reconstruction can be met using a magnet in air. The only dead material along the muon path is given by the conductors needed to generate the magnetic field and the position detectors instrumenting the magnet itself. For the conductors the use of aluminum instead of copper is preferable due to the lower $Z$ and a density lower by a factor 3. The magnetic field needed in the low momentum range covered by the ACM is in the range $0.1-0.15 \mathrm{~T}$. A spatial resolution in the range of $0.1-1 \mathrm{~mm}$ can be reached using drift tubes [49] as high precision trackers in combination with scintillator strip detectors [50]. These could provide the external trigger needed by the drift tubes and a coarse spatial measurement in the nonbending direction, that is, the direction parallel to the drift tubes. Silicon photomultiplier (SiPM) devices may eventually be used to read out some scintillator planes embedded in the magnetic field.

The general layout of the two OPERA [51] iron spectrometers fulfills the requirements of the ICM detector, and they could eventually be used for the CERN project putting the two magnets one after the other in order to obtain a total of $4 \mathrm{~m}$ longitudinal thickness of iron.

The OPERA spectrometers are built assembling vertical iron plates (slabs) in a planar structure of $875 \mathrm{~cm}$ (width) $\times 800 \mathrm{~cm}$ (height). Each passive plane is made out of seven adjacent iron slabs. Resistive Plate Chambers (RPC) [52] are sandwiched between iron planes; 21 RPC detectors, arranged in seven rows and three columns, are used in each active plane. Each RPC detector has a rectangular shape and covers an area of about $3.2 \mathrm{~m}^{2}$. The RPCs provide tracking measurements with about $1 \mathrm{~cm}$ resolution using the digital read-out of strips with a $2.6 \mathrm{~cm}$ pitch in the bending direction and $3.5 \mathrm{~cm}$ in the nonbending direction. The magnet is made of two arms with 22 RPC layers alternating with iron layers. Top and bottom iron yokes are connecting the arms. Copper coils surrounding the yokes are used to generate a magnetic field of about $1.5 \mathrm{~T}$ in the iron circuit. A total of 924 RPC chambers are needed to instrument each spectrometer. They could be recovered from the OPERA spectrometers and reused. These RPC chambers are standard $2 \mathrm{~mm}$ gap 

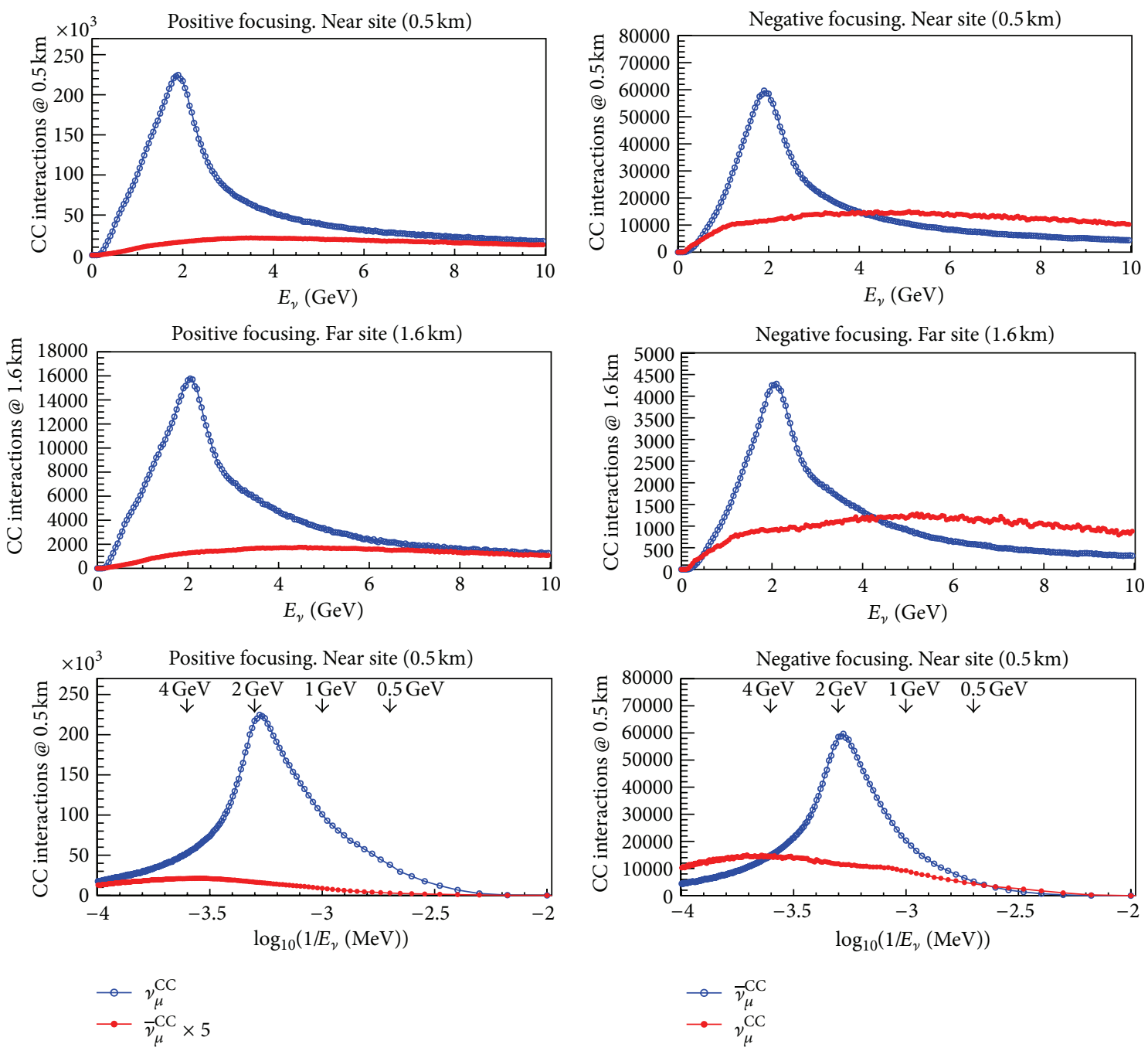

(a)

(b)

FIGURE 1: Expected neutrino CC interactions in the no-oscillation hypothesis for positive polarity (a) and negative polarity (b) for the new proposed CERN neutrino beam (elaborated from [46]) and for an integrated luminosity of 1 year. In the first (second) row the rates are shown at the near (far) position. In the third row the rates expected at the near site are displayed as function of $\log _{10}(1 / E)$, used in the paper (see Section 3). Note that the distributions of the antineutrino rates in case of positive polarity are multiplied by a factor 5 to allow a better visual inspection.

chambers with bakelite electrodes with resistivity in the range from $10^{11}$ to $5 \times 10^{12} \Omega \cdot \mathrm{cm}$ at $T=20^{\circ} \mathrm{C}$. In OPERA they are operated in the streamer regime with a gas mixture made of $\mathrm{Ar} / \mathrm{C}_{2} \mathrm{H}_{2} \mathrm{~F}_{4} / \mathrm{I}-\mathrm{C}_{4} \mathrm{H}_{10} / \mathrm{SF}_{6}$ in the volume ratios of 75.4/20/4/0.6 at five refills/day. The high-amplitude streamer signals produced by charged tracks crossing the gas volume allow to house the front-end discriminators in racks placed on top of the spectrometer.

\section{The $v_{\mu}$ Disappearance Analysis}

The use of at least two sites where neutrino interactions can occur is mandatory to observe the oscillation pattern, as established by almost all the most recent neutrino experiments. Indeed the disappearance probability due to an additional sterile neutrino is given by the usual twoflavour formula:

$$
P\left(v_{\alpha} \longrightarrow v_{\alpha}\right)=1-\sin ^{2}(2 \theta) \cdot \sin ^{2}\left(1.267 \cdot \Delta m^{2} \cdot \frac{L}{E}\right),
$$

$$
\text { ( } L \text { in } \mathrm{km}, E \text { in } \mathrm{GeV} \text { ), }
$$

where the disappearance of flavour $\alpha$ is due to the oscillation of neutrino mass states at the $\Delta m^{2}$ scale and at an effective mixing angle $\theta$ that can be simply parametrized as a function of the elements of a $3+1$ extended mixing matrix. As $L$ is fixed by the experiment location, the oscillation is naturally driven by the neutrino energy, with an amplitude determined by the mixing parameter.

The disappearance of muon neutrinos due to the presence of an additional sterile state depends only on terms of the 


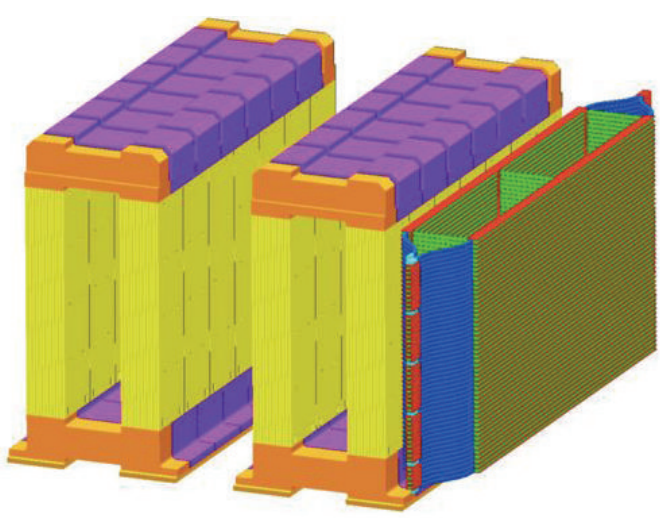

FIgURE 2: Sketch of the far site spectrometer that could be built by extensively reusing materials available from the OPERA spectrometers [51]. The basic elements of the new ACM concept are also depicted (courtesy of the NESSiE Collaboration). Neutrinos are traveling from right to left.

extended PMNS $[53,54]$ mixing matrix $\left(U_{\alpha i}\right.$ with $\alpha=e$, $\mu, \tau$ and $i=1, \ldots, 4)$ involving the $\nu_{\mu}$ flavor state and the additional mass eigenstate 4 . In a $3+1$ model at Short Baseline (SBL) we have

$$
P\left(\nu_{\mu} \longrightarrow v_{\mu}\right)_{\mathrm{SBL}}^{3+1}=1-4\left|U_{\mu 4}\right|^{2}\left(1-\left|U_{\mu 4}\right|^{2}\right) \sin ^{2} \frac{\Delta m_{41}^{2} L}{4 E} .
$$

In contrast, appearance channels (i.e. $v_{\mu} \rightarrow v_{e}$ ) are driven by terms that mix up the couplings between the initial and final flavour states and the sterile state yielding a more complex picture:

$$
P\left(\nu_{\mu} \longrightarrow v_{e}\right)_{\mathrm{SBL}}^{3+1}=4\left|U_{\mu 4}\right|^{2}\left|U_{e 4}\right|^{2} \sin ^{2} \frac{\Delta m_{41}^{2} L}{4 E} .
$$

This also holds in extended $3+n$ models.

It is interesting to notice that the appearance channel is suppressed by two more powers in $\left|U_{\alpha 4}\right|$. Furthermore, since $\nu_{e}$ or $\nu_{\mu}$ appearance requires $\left|U_{e 4}\right|>0$ and $\left|U_{\mu 4}\right|>0$, it should be naturally accompanied by a corresponding $\nu_{e}$ and $v_{\mu}$ disappearance. In this sense the disappearance searches are essential for providing severe constraints on the models of the theory (a more extensive discussion on this issue can be found, e.g., in Section 2 of [42]).

It must also be noted that the number of $v_{e}$ neutrinos depends on the $\nu_{e} \rightarrow v_{s}$ disappearance and $\nu_{\mu} \rightarrow v_{e}$ appearance and, naturally, from the intrinsic $\nu_{e}$ contamination in the beam. On the other hand, the amount of $\nu_{\mu}$ neutrinos depends only on the $\nu_{\mu} \rightarrow \nu_{s}$ disappearance and $\nu_{e} \rightarrow v_{\mu}$ appearance, but the latter is much smaller due to the fact that the $\nu_{e}$ contamination in $\nu_{\mu}$ beams is usually at the percent level. Therefore in the $\nu_{\mu}$ disappearance channel the oscillation probabilities in both near and far detectors can be measured without any interplay of different flavours, that is, by the same probability amplitude.

By taking into account the lengths $L$ of the locations given in the CERN proposal, $L_{\text {Near }}=460 \mathrm{~m}$ and $L_{\mathrm{Far}}=1600 \mathrm{~m}$, we may plot the disappearance probability as a function of the more convenient variable $\log _{10}(1 / E)$, using $E$ in $\mathrm{MeV}$ to avoid singularities. As an example, in Figure 3 the disappearance probability is shown for the near and far sites, by assuming an amplitude that corresponds to the averaged reactor anomaly $\bar{\nu}_{e}$ disappearance, $R=0.927 \pm 0.023[12-14]$ and $\Delta m^{2}=1 \mathrm{eV}^{2}$ (it is worthwhile to note that the disappearance amplitude affects the sensitivity to the mixing angle that in turn depends mainly on the statistical extent of the data. Therefore the same evidence can be obtained for smaller amplitudes, that is, smaller effective mixing angles, by increasing the data sample. Finally the ultimate limit of an experiment is set by the intrinsic systematic errors corresponding to the kinematics of the neutrino interaction and to the muon reconstruction sensitivity. Figure 8 below illustrates the issue). It appears that at $\Delta m^{2} \approx 1 \mathrm{eV}^{2}$ oscillations are already visible at the near site. Such a behaviour may turn out to help the actual measurement by plotting the ratio between the far and near sites data as a function of $\log _{10}(1 / E)$ (Figure 4$)$. The Far/Near ratio of the oscillation probabilities well illustrates the pattern that could be observed at the CERN ShortBaseline experiment: a decrease above $1 \mathrm{GeV}$ and an increase below $1 \mathrm{GeV}$ of the neutrino energy!

The disappearance patterns have to be evaluated after folding cross-sections and efficiencies with the neutrino fluxes and detector geometry. The Far/Near pattern does not depend at first order either on cross-sections and efficiencies or on the fluxes as far as they are similar at the two sites and within the use of two identical detector systems. However, due to the different $\nu$ and $\bar{\nu}$ cross-sections, errors are enhanced when neutrinos and antineutrinos are not identified and separated on an event-by-event basis. Therefore the use of magnetic detectors is mandatory to keep systematics under control. Then the largest source of error consists of the smearing introduced by the reconstruction procedure. Eventually this issue will be addressed by considering a conservative systematic effect in the muon momentum reconstruction.

Figure 5(a) illustrates a realistic observation of the nonoscillation probability when convoluted with the estimated CERN $\nu_{\mu}$ beam flux and the neutrino cross-sections, by using the detector systems described above and one year of data taking. The flatness is lost as expected. However a specific case of oscillation is drawn, confirming that the workable behaviour is still present. The correlated estimator, the double ratio parameter, defined as (Far/Near) $)_{\text {oscillated }} /(\text { Far/Near })_{\text {unoscillated }}$, is also shown in the bottom of the figure. It can be used for the statistical analysis. In the following we will demonstrate how the oscillation behaviour of the Far/Near parameter will allow an increase of an order of magnitude in the sensitivity to the mixing angle, taking into account the systematic errors, too.

Another parameter can be used in case measurement of Neutral Current (NC) events becomes available, either with the proposed LAr detectors or even with a less refined and massive detector. The NC/CC ratio becomes then a valuable estimator [43] that may enter into the evaluation of the significance of the sterile neutrino observation. A study that 


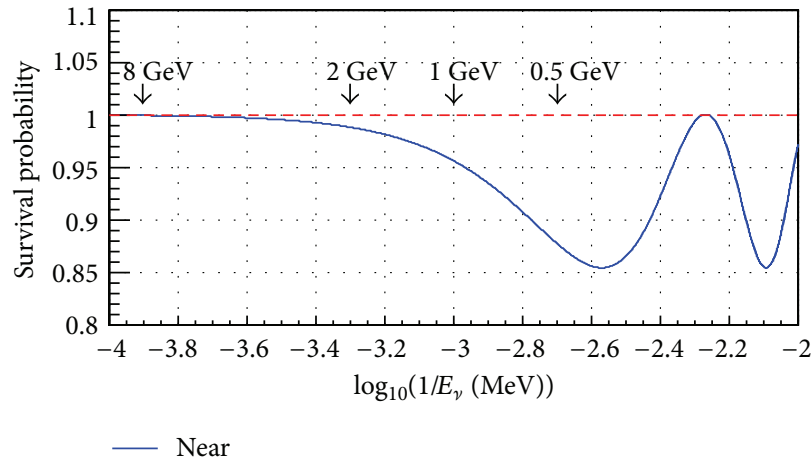

(a)

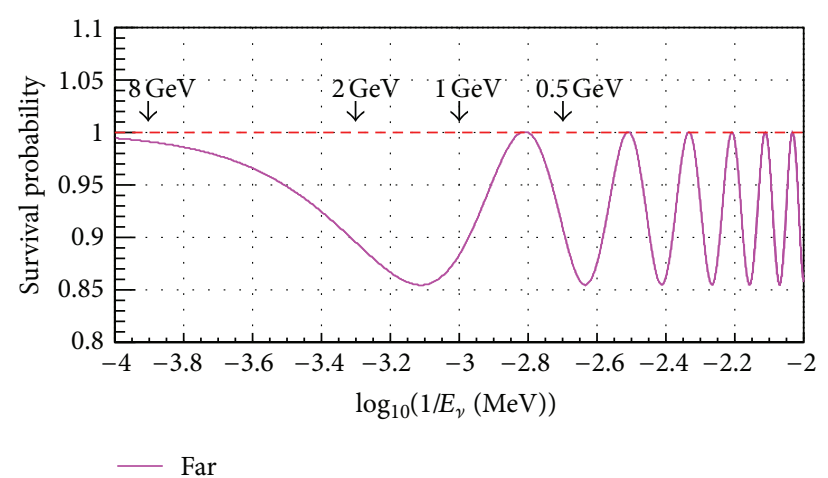

(b)

FIGURE 3: Disappearance probabilities in the two-flavour limit at near (a) and far (b) sites, at 460 and $1600 \mathrm{~m}$, respectively, by using the amplitude provided by the reactor anomaly, 0.146 , and the mass scale $\Delta m^{2}=1 \mathrm{eV}^{2}$. The $x$-axis corresponds to $\log _{10}\left(1 / E_{v}\right)$, with $E$ in $\mathrm{MeV}$.

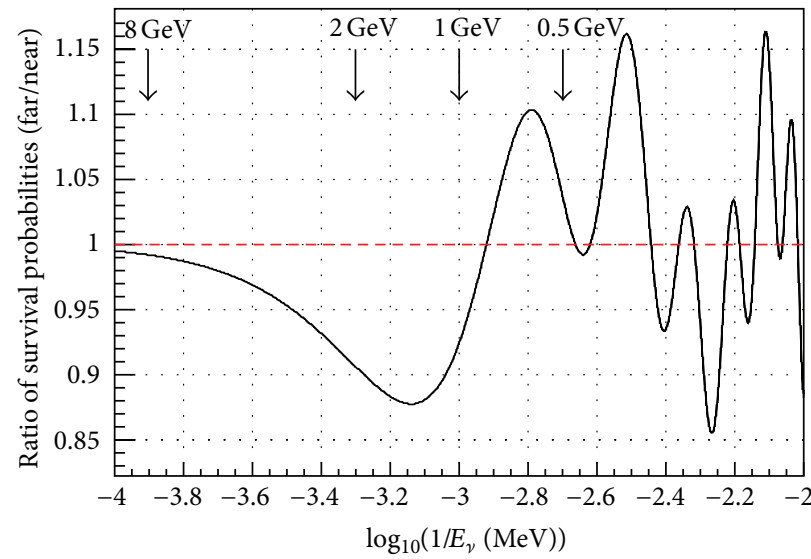

FIGURE 4: The ratio of the disappearance probabilities at near and far sites, 460 and $1600 \mathrm{~m}$, respectively, in the two-flavour limit, by using the amplitude provided by the reactor anomaly, 0.146 , and the mass scale $\Delta m^{2}=1 \mathrm{eV}^{2}$. The $x$-axis corresponds to $\log _{10}\left(1 / E_{v}\right)$, with $E$ in $\mathrm{MeV}$.

includes the information from Neutral Current events is out of the scope of the present paper. However for completeness we would like to briefly outline here the issue as it was originally described in [43].

The observation of a depletion in NC events would be a direct and manifest signature of the existence of sterile neutrinos. In fact the NC event rates are unaffected by standard neutrino mixing being flavour-blind such that their disappearance could only be explained by $\nu_{\mu}\left(\nu_{e}\right) \rightarrow v_{s}$ transitions. Even if NC events, either from $\nu_{e}$ or $\nu_{\mu}$, are efficiently detected by a Liquid Argon detector or a less refined detector, the transition rate measured with $\mathrm{NC}$ events has to agree with the $\nu_{\mu}$ CC disappearance rate once the $\nu_{\mu} \rightarrow \nu_{\tau}$ and $\nu_{\mu} \rightarrow \nu_{e}$ contributions have been subtracted. Therefore the NC disappearance is measured at best by the double ratio: $(\mathrm{NC} / \mathrm{CC})_{\mathrm{Far}} /(\mathrm{NC} / \mathrm{CC})_{\mathrm{Near}}$. The double ratio is the most robust experimental quantity to detect $\mathrm{NC}$ disappearance, once $\mathrm{CC}_{\mathrm{Near}}$ and $\mathrm{CC}_{\mathrm{Far}}$ are precisely measured thanks to the spectrometers, at the near and far locations. For that it is mandatory to disentangle $\nu_{\mu}$ and $\bar{\nu}_{\mu}$ contributions.

\section{Simulation and Results}

The magnetic detector system that has to be developed for the $\nu_{\mu}$ disappearance measurement should take into account all the considerations depicted in previous sections. The system developed by the NESSiE Collaboration [43] is actually well suited since it couples a very powerful high- $Z$ magnet for the momentum measurement via range to a low- $Z$ magnet, to extend the useful muon momentum interval as low as possible, to allow charge discrimination on an event-by-event basis, and to allow NC event measurement whether coupled to an adequate (but not necessarily highly performant and with large mass) detector to identify NC events.

In the following we concentrate on the results achievable by the Far/Near estimators described in the previous section, by exploiting a full neutrino beam simulation, an up-to-date neutrino interaction model (GENIE 2.6 [55]), with detailed interactions in low- $Z$ and high- $Z$ magnets and a generic geometry. The Iron Core Magnets have been simulated by a massive cube of iron with a mass equivalent to the OPERA 


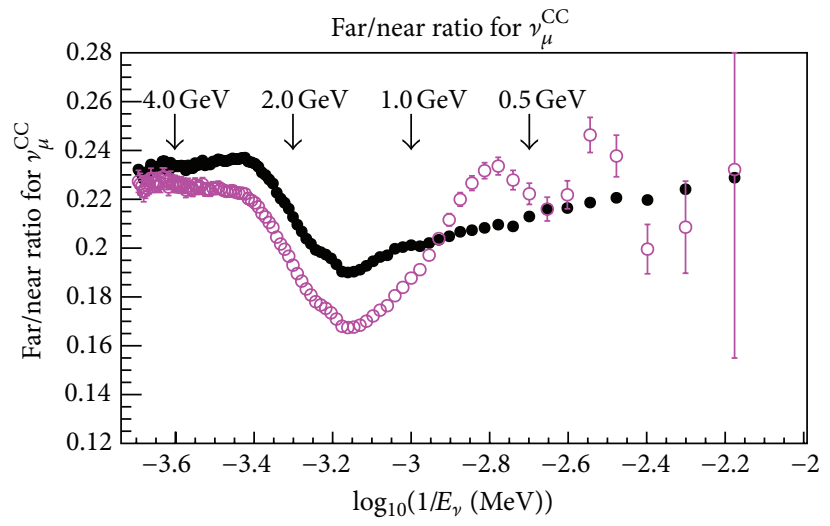

$\rightarrow$ No oscillations

$\rightarrow$ Oscillations

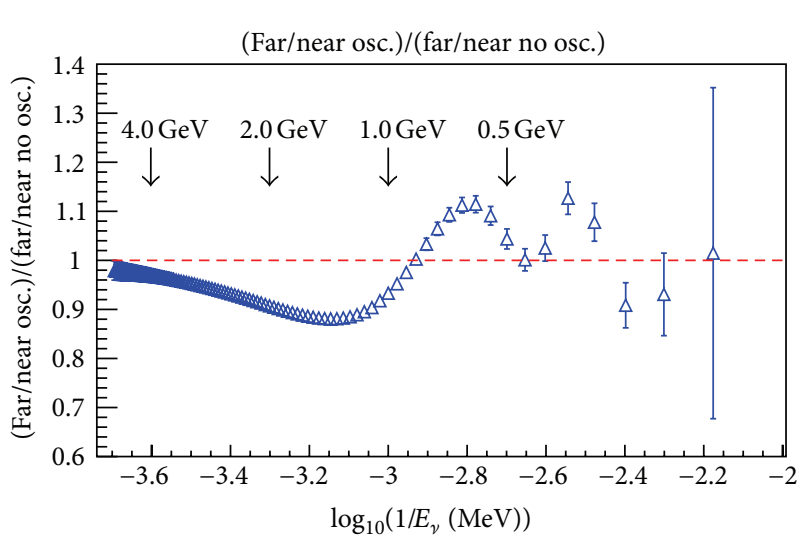

(b)

FIGURE 5: The oscillated and unoscillated $\nu_{\mu}$ event distributions, parametrized in $\log _{10}\left(1 / E_{\gamma}\right)$, for a luminosity of $4.5 \times 10^{19}$ p.o.t. of the CERN $\nu_{\mu}$ beam flux over the two-magnet system described in the text, are plotted with their statistical errors. (a) The far/near collected ratios are drawn. The no-oscillation shape is due to the convolution of the neutrino cross-section and the fluxes at the near and far site, while the oscillated shape is due to the sterile inclusion (with $\Delta m^{2}=1 \mathrm{eV}^{2}$ and mixing amplitude equal to 0.146 ). (b) The double ratio (Far/Near) $)_{\text {oscillated }} /(\text { Far/Near })_{\text {unoscillated }}$ is shown for the same sterile assumption.

magnets and by using a 90\% inner fiducial volume (770 tons and 330 tons for the far and near locations, resp.). Single hits have been extracted at the position of the RPC detectors and a realistic resolution has been included $(0.75 \mathrm{~cm}$ for the single hit). The ACM has been simulated as an empty $1 \mathrm{~m}$ deep region with $0.1 \mathrm{~T}$ magnetic field, limited by two vertical aluminum slabs. The overall simulation corresponds to the GLoBES software [56] (version 3.0.11), to which it was partially compared and cross checked.

The muon reconstruction requires a minimal penetration length that corresponds to a $0.5 \mathrm{GeV}$ cut. A conservative resolution of $10 \%$ was used for the momentum evaluation, while the charge misidentification was fixed at the $1 \%$ level. Systematic effects due to miscalibration of the detectors have been conservatively taken at $0.5 \%$ and $1 \%$ levels, which include systematics due to the relative flux at the near and far positions.

In Figure 6 the double ratio Far/Near is shown as obtained by using the reconstructed muon momentum, for different values of $\Delta m^{2}$ scales. The relevant behaviour of the estimator is confirmed for $\Delta m^{2}$ values above $\approx 2 \mathrm{eV}^{2}$ also in this very realistic and conservative approach. In fact, by using the muon reconstructed momentum in CC events instead of the true $\nu_{\mu}$ energy, the oscillations properties will be affected by the kinematics and the interaction processes (it is worthwhile to note that there are mostly quasi-elastic interactions in the accounted-for energy range).

Finally, in Figure 7 the estimated limits at 95\% C.L. on $\nu_{\mu}$ disappearance that can be achieved via the Far/Near estimator are shown for different data periods (3, 5, and 10 years, corresponding to $13.5 \cdot 10^{19}, 22.5 \cdot 10^{19}$, and 45.0 . $10^{19}$ p.o.t., resp.). The different results for $\nu_{\mu}$ and $\bar{\nu}_{\mu}$ beams were evaluated using the two variables, $p$ and $\log _{10}(1 / p)$. In negative polarity runs the muon charge identification allows an independent, simultaneous, and similar-sensitivity measurements of the $\bar{\nu}_{\mu}$ and $\nu_{\mu}$ disappearance rates, due to the large $\nu_{\mu}$ contamination in the $\bar{\nu}_{\mu}$ beam.

The parameterization used for the neutrino energy, $\log _{10}(1 / E)$, which we conservatively prefer to address as $\log _{10}\left(1 / p_{\mu \text {-rec }}\right)$, provides slightly better limits in almost all the $\left(\Delta m^{2}, \sin ^{2}(2 \theta)\right)$ excluded regions. This is partially due to the Gaussian shape sensitivity of $1 / p$ when cuts are applied to the corresponding variable. Moreover, the elucidation of the behaviour of the Far/Near ratio in terms of depletions and excesses in different regions of the spectra allows a better comparison between unoscillated and oscillated hypotheses.

As a result more than an order of magnitude improvement can be obtained in the sensitivity to the mixing parameter space between standard neutrino and sterile ones with respect to today's limits over the whole $\Delta m^{2}$ range investigated. Specifically the mixing angle sensitivity (at $95 \%$ C.L.) reaches $2.2 \times 10^{-3}$ at $\Delta m^{2}=1 \mathrm{eV}^{2}$, while at full mixing a $\Delta m^{2}=0.03 \mathrm{eV}^{2}$ sensitivity at $95 \%$ C.L. is obtained already with only 3 years of data collection with the neutrino beam.

Slightly worse results are obtained by collecting data with a negative polarity beam. The limiting mixing angle sensitivity at $95 \%$ C.L. is around $10^{-2}$ at $\Delta m^{2}=1 \mathrm{eV}^{2}$. At full mixing a $\Delta m^{2}=0.1 \mathrm{eV}^{2}$ sensitivity at $95 \%$ C.L. is obtained with 3 years of data collection with the antineutrino beam, the limiting factor being the intensity of the $\bar{\nu}_{\mu}$ beam. However it is worthwhile to note that by operating in negative polarity similar sensitivities can be obtained at the same time also for the neutrino component due to the large contamination of $v$ in the anti- $\nu$ beams. 


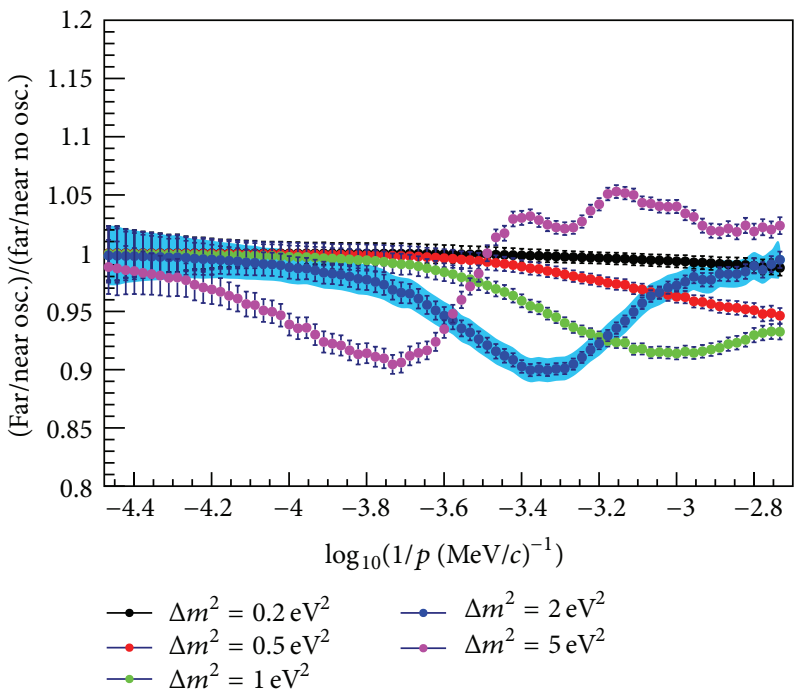

Figure 6: The double ratio of the disappearance probabilities at the near and far sites, 460 and $1600 \mathrm{~m}$, respectively, by using the amplitude provided by the reactor anomaly, 0.146 , and mass scales $\Delta m^{2}$ ranging from 0.1 to $5 \mathrm{eV}^{2}$ as a function of $\log _{10}\left(1 / p_{\mu}\right)$, where " $p$ " is the reconstructed muon momentum in $\mathrm{MeV} / \mathrm{c}$. We apply cuts on the minimum muon path length $(25 \mathrm{~cm}$ of range in iron) and the minimum momentum $(500 \mathrm{MeV} / \mathrm{c})$. A $90 \%$ fiducial acceptance of the magnet volume and an uncorrelated 1\% systematic error (from OPERA experience [57]) are also considered. Data collection corresponds to 1 year, that is, $4.5 \cdot 10^{19}$ p.o.t. The error bars correspond to the statistical errors. The blue band for $\Delta m^{2}=2 \mathrm{eV}^{2}$ is obtained by including the systematic error summed quadratically to the statistical one, the same result being obtained for the other mass scales.

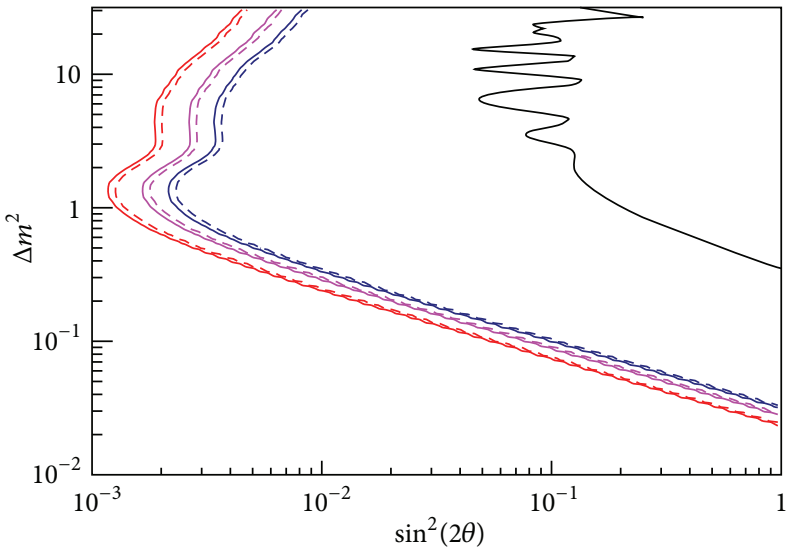

$\begin{array}{ll}-3 \text { years, } \log (1 / p) \text { estimator } & ---3 \text { years, } p \text { estimator } \\ -5 \text { years, } \log (1 / p) \text { estimator } & ---5 \text { years, } p \text { estimator } \\ -10 \text { years, } \log (1 / p) \text { estimator } & ---10 \text { years, } p \text { estimator }\end{array}$

(a)

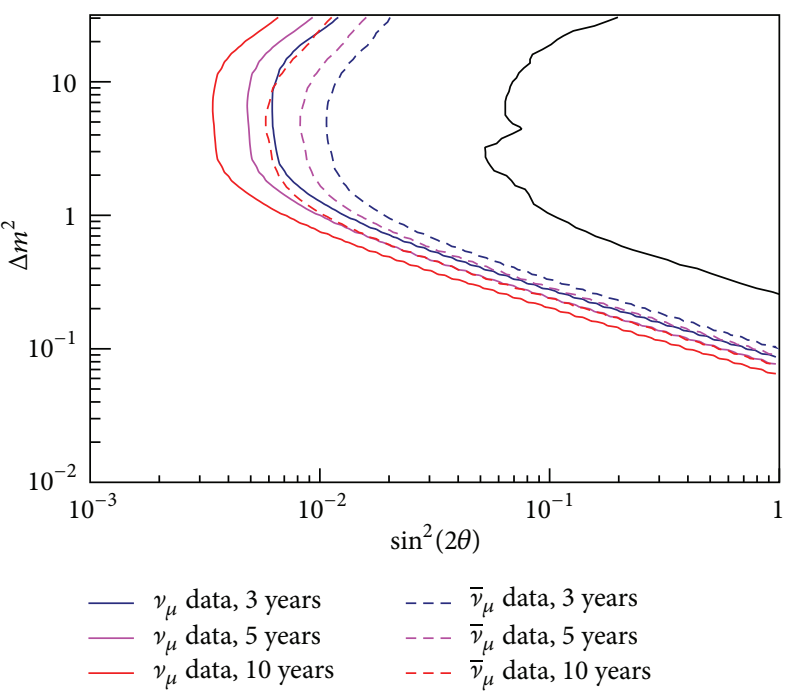

(b)

FIGURE 7: The estimated limits at 95\% C.L. for $v_{\mu}$ disappearance at a Short-Baseline beam at CERN for several luminosity running periods and different beam polarities, with a two-site massive spectrometer (770 tons and 330 tons, resp.) with $90 \%$ inner fiducial volume. (a) Refers to the positive polarity beam. The continuous (dashed) lines correspond to the sensitivity limits obtained with the $\log _{10}(1 / p)(p)$ variable. 3 years correspond to $13.5 \cdot 10^{19}$ p.o.t., 5 years to $22.5 \cdot 10^{19}$ p.o.t., and 10 years to $45.0 \cdot 10^{19}$ p.o.t. The exclusion limit from combined MiniBooNE and SciBooNE $v_{\mu}$ disappearance result at $90 \%$ C.L. from [33] is shown for comparison by the black curve in the right. (b) Refers to the negative polarity beam. Sensitivity limits are evaluated with the $\log _{10}(1 / p)$ variable. Clearly the negative polarity run allows the contemporary analysis of the $\bar{\nu}_{\mu}$ and $\nu_{\mu}$ disappearance exclusion regions thanks to the disentangling of the muon charge on an event-by-event basis. The black curve in the right shows for comparison the central value of the sensitivity at 90\% C.L. from combined MiniBooNE and SciBooNE $\bar{\nu}_{\mu}$ disappearance result [34]. 


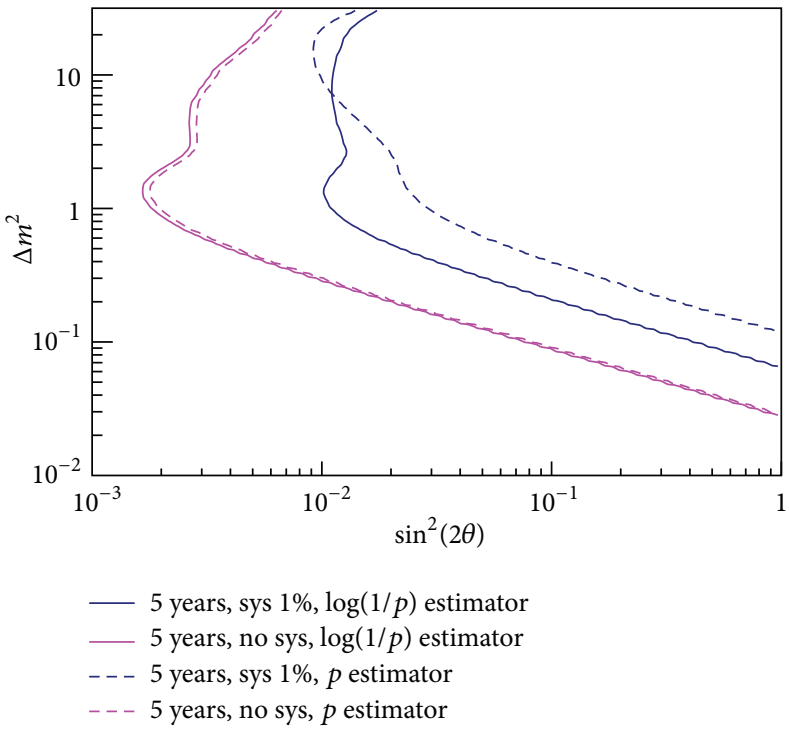

(a)

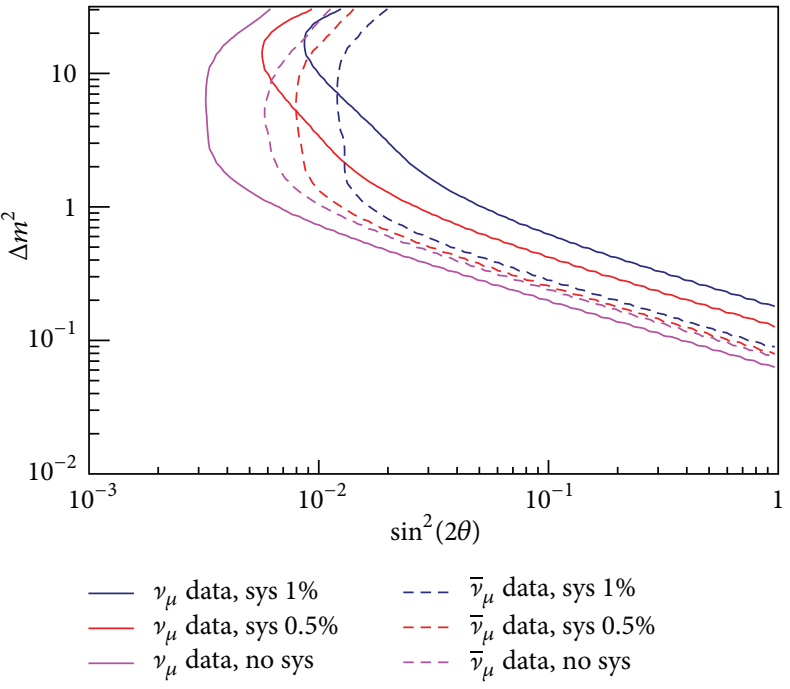

(b)

FIGURE 8: Systematic effects for the estimated limits at 95\% C.L. for $v_{\mu}$ disappearance at a Short-Baseline beam at CERN for 5 (positive polarity, (a)) and 10 (negative polarity, (b)) years of data taking, with a two-site massive spectrometer (770 tons and 330 tons, resp.) with $90 \%$ inner fiducial volume. The systematic uncertainty, corresponding to each of the exclusion contours, ranging from $0 \%$ to $1 \%$, is indicated in the legend as "sys". The exclusion regions are evaluated by using the $\log _{10}(1 / p)$ (continuous lines) and $p$ (dashed lines) variables in case of the positive polarity beam data taking.

The relevance of statistics in presence of systematic effects is depicted in Figure 8. It is evident that the overall error, dominated by the sensitivity on the measurement of the muon momentum, puts an intrinsic limitation to the data statistics which can be collected. As expected the useful luminosity that can be collected is asymptotically limited by systematics. The positive effect of the variable $\log _{10}\left(1 / p_{\mu \text {-rec }}\right)$ is more evident when larger errors are in place.

\section{Conclusions}

Neutrino physics is receiving more and more attention as a venue for the long standing search for new physics beyond the Standard Model. The current anomalies which do not fit into the established standard scenario with 3 neutrinos deserve refined studies and experiments. The CERN proposal for a new Short-Baseline experimental project is a very valuable one. We illustrated the current critical tensions in the muon neutrino disappearance field and the achievements that can be obtained within the CERN project. Specifically, by considering $\mathcal{O}(1)$ kton massive spectrometers, an improvement by an order of magnitude can be obtained in the sensitivity to the mixing parameter space between standard neutrinos and sterile ones with respect to today's limits. Conversely, a possible $v_{\mu}$ disappearance signal will be essential to measure the relevant physical parameters and to fully disentangle the different sterile models.

An effective analysis can be performed with a two-site experiment by using muon spectrometers with a low- $Z$ part that allows clean charge identification on an event-by-event basis and with a massive part allowing clean momentum measurement through range. Such a kind of spectrometer is under study by the NESSiE Collaboration and might be available with a limited investment. The performances of these kinds of detectors, enlightened by the type of analysis developed in this paper, are suitable to put a definitive result on the sterile neutrino issue at the $\mathrm{eV}$ mass scale.

\section{Acknowledgments}

The authors are heartily dependent of the contributions of the NESSiE, ICARUS, and CERN-CENF groups in developing the CERN project and the experimental proposals. The authors wish also to warmly thank the encouragements of Marzio Nessi and Carlo Rubbia in supporting such studies. The authors are finally in debt to Maury Goodman for suggested criticisms and a careful proof-reading of the paper.

\section{References}

[1] F. P. An, J. Z. Bai, A. B. Balantekin et al., "Observation of electron-antineutrino disappearance at Daya Bay", Physical Review Letters, vol. 108, no. 17, Article ID 171803, 7 pages, 2012.

[2] J. Ahn, S. Chebotaryov, J. H. Choi et al., "Observation of reactor electron antineutrino disappearance in the RENO experiment," Physical Review Letters, vol. 108, no. 19, Article ID 191802, 6 pages, 2012.

[3] Y. Abe, C. Aberle, T. Akiri et al., "Indication of reactor $\bar{v}_{e}$ disappearance in the double chooz experiment," Physical Review Letters, vol. 108, Article ID 131801, 7 pages, 2012.

[4] K. Abe, N. Abgrall, Y. Ajima et al., "Indication of electron neutrino appearance from an accelerator-produced off-axis 
muon neutrino beam," Physical Review Letters, vol. 107, no. 4, Article ID 041801, 8 pages, 2011.

[5] B. Pontecorvo, "Neutrino experiments and the problem of conservation of leptonic charge," Zhurnal Èksperimental'noi $i$ Teoreticheskoi Fiziki, vol. 53, p. 1717, 1967, translation in Soviet Physics-Journal of Experimental and Theoretical Physics, vol. 26, p. 984, 1968.

[6] K. N. Abazajian, M. A. Acero, S. K. Agarwalla et al., "Light sterileneutrinos: a white paper," http://arxiv.org/abs/1204.5379.

[7] A. Aguilar, L. B. Auerbach, R. L. Burman et al., "Evidence for neutrino oscillations from the observation of $\bar{v}_{e}$ appearance in a $\bar{v}_{\mu}$ beam," Physical Review D, vol. 64, no. 11, Article ID 112007, 22 pages, 2001.

[8] A. A. Aguilar-Arevalo, A. O. Bazarko, S. J. Brice et al., "Search for electron neutrino appearance at the $\Delta m^{2} \sim 1 \mathrm{eV}^{2}$ scale," Physical Review Letters, vol. 98, no. 23, Article ID 231801, 7 pages, 2007.

[9] A. A. Aguilar-Arevalo, C. E. Anderson, S. J. Brice et al., "Event excess in the MiniBooNE search for $\bar{\nu}_{\mu} \rightarrow \bar{\nu}_{e}$ oscillations," Physical Review Letters, vol. 105, no. 18, Article ID 181801, 2010.

[10] C. C. Polly, "Improved statistics in the search for $\bar{\nu}_{\mu} \rightarrow \bar{\nu}_{e}$ oscillations in MiniBooNE," Nuclear Physics B, vol. 235-236, no. 1, pp. 207-213, 2013.

[11] A. Aguilar-Arevalo, B. C. Brown, L. Bugel et al., "Improved search for $\bar{v}_{\mu} \rightarrow \bar{v}_{e}$ oscillations in the MiniBooNE experiment," Physical Review Letters, vol. 110, Article ID 161801, 6 pages, 2013.

[12] G. Mention, M. Fechner, Th. Lasserre et al., "Reactor antineutrino anomaly," Physical Review D, vol. 83, Article ID 073006, 20 pages, 2011.

[13] D. Lhuillier, "Reactor flux calculations," Nuclear Physics B, vol. 235-236, pp. 11-16, 2013.

[14] T. Lasserre, "Testing the reactor and gallium anomalies with intense (anti)neutrino emitters," Nuclear Physics B, vol. 235-236, pp. 214-219, 2013.

[15] J. N. Abdurashitov, V. N. Gavrin, V. V. Gorbachev et al., "Measurement of the solar neutrino capture rate with gallium metal. III. Results for the 2002-2007 data-taking period," Physical Review C, vol. 80, no. 1, Article ID 015807, 16 pages, 2009.

[16] J. N. Abdurashitov, V. N. Gavrin, S. V. Ginn et al., “The RussianAmerican Gallium Experiment (SAGE) Cr neutrino source measurement," Physical Review Letters, vol. 77, no. 23, pp. 47084711, 1996.

[17] J. N. Abdurashitov, V. N. Gavrin, S. V. Girin et al., "Measurement of the response of a gallium metal solar neutrino experiment to neutrinos from a ${ }^{51} \mathrm{Cr}$ source," Physical Review C, vol. 59, pp. 2246-2263, 1999.

[18] J. N. Abdurashitov, V. N. Gavrin, S. V. Girin et al., "Measurement of the response of a Ga solar neutrino experiment to neutrinos from a ${ }^{37}$ Ar source," Physical Review C, vol. 73, Article ID 045805, 12 pages, 2006.

[19] F. Kaether, W. Hampel, G. Heusser, J. Kiko, and T. Kirsten, "Reanalysis of the Gallex solar neutrino flux and source experiments," Physics Letters B, vol. 685, no. 1, pp. 47-54, 2010.

[20] P. Anselmann, R. Fockenbrock, W. Hampel et al., "First results from the ${ }^{51} \mathrm{Cr}$ neutrino source experiment with the GALLEX detector," Physics Letters B, vol. 342, pp. 440-450, 1995.

[21] W. Hampel, G. Heusser, J. Kiko et al., "Final results of the ${ }^{51} \mathrm{Cr}$ neutrino source experiments in GALLEX," Physics Letters B, vol. 420, no. 1-2, pp. 114-126, 1998.

[22] C. Giunti and M. Laveder, "Statistical significance of the gallium anomaly," Physical Review C, vol. 83, no. 6, Article ID 065504, 2011.
[23] J. Kopp, M. Maltoni, and T. Schwetz, "Are there sterile neutrinos at the eV scale?" Physical Review Letters, vol. 107, no. 9, Article ID 091801, 2011.

[24] C. Giunti and M. Laveder, "3+1 and 3+2 sterile neutrino fits," Physical Review D, vol. 84, no. 7, Article ID 073008, 10 pages, 2011.

[25] E. Kuflik, S. D. McDermott, and K. M. Zurek, "Neutrino phenomenology in a 3+1+1 framework," Physical Review D, vol. 86, no. 3, Article ID 033015, 2012.

[26] A. E. Nelson, "Effects of CP violation from neutral heavy fermions on neutrino oscillations, and the LSND/MiniBooNE anomalies," Physical Review D, vol. 84, no. 5, Article ID 053001, 2011.

[27] J. M. Conrad, C. M. Ignarra, G. Karagiorgi, M. H. Shaevitz, and J. Spitz, "Sterile neutrino fits to short-baseline neutrino oscillation measurements," Advances in High Energy Physics, vol. 2013, Article ID 163897, 26 pages, 2013.

[28] J. Kopp, P. A. N. Machado, M. Maltoni, and T. Schwetz, "Sterile neutrino oscillations: the global picture," Journal of High Energy Physics, vol. 2013, article 050, 2013.

[29] T. Schwetz, "Status of sterile neutrino oscillations," Nuclear Physics B, vol. 235-236, pp. 229-235, 2013.

[30] C. Giunti, M. Laveder, Y. F. Li, Q. Y. Liu, and H. W. Long, "Update of short-baseline electron neutrino and antineutrino disappearance," Physical Review D, vol. 86, no. 11, Article ID 113014, 14 pages, 2012.

[31] F. Dydak, G. J. Feldman, C. Guyot et al., "A search for $v_{\mu}$ oscillations in the $\Delta m^{2}$ range $0.3-90 \mathrm{eV}^{2}$," Physics Letters $B$, vol. 134, p. 281, 1984.

[32] A. A. Aguilar-Arevalo, C. E. Anderson, A. O. Bazarko et al., "Search for muon neutrino and antineutrino disappearance in MiniBooNE," Physical Review Letters, vol. 103, no. 6, Article ID 061802, 5 pages, 2009.

[33] K. B. M. Mahn, Y. Nakajima, A. A. Aguilar-Arevalo et al., "Dual baseline search for muon neutrino disappearance at $0.5 \mathrm{eV}^{2}<$ $\Delta m^{2}<40 \mathrm{eV}^{2}$," Physical Review D, vol. 85, no. 3, Article ID 032007, 10 pages, 2012.

[34] G. Cheng, W. Huelsnitz, A. A. Aguilar-Arevalo et al., "Dual baseline search for muon antineutrino disappearance at $0.1 \mathrm{eV}^{2}<\Delta m^{2}<100 \mathrm{eV}^{2}$," Physical Review D, vol. 86, no. 5, Article ID 052009, 14 pages, 2012.

[35] P. Adamson, C. Andreopoulos, D. J. Auty et al., "Search for sterile neutrino mixing in the MINOS long-baseline experiment," Physical Review D, vol. 81, no. 5, Article ID 052004, 2010.

[36] S. Razzaque and A. Y. Smirnov, "Searches for sterile neutrinos with IceCube DeepCore," Physical Review D, vol. 85, Article ID 093010, 2012.

[37] A. Esmaili, F. Halzen, and O. Peres, "Constraining sterile neutrinos with AMANDA and IceCube atmospheric neutrino data," Journal of Cosmology and Astroparticle Physics, vol. 2012, no. 11, article 041, 2012.

[38] A. Palazzo, "Phenomenology of light sterile neutrinos: a brief review," Modern Physics Letters A, vol. 28, no. 7, 11 pages, 2013.

[39] P. Huber, "On the determination of anti-neutrino spectra from nuclear reactors," Physical Review C, vol. 84, no. 2, Article ID 024617, 16 pages, 2011.

[40] P. Huber, "Reactor neutrinos fluxes," in Proceedings of the 15th International Workshop on Neutrino Telescopes (NeuTel '13), Venice, Italy, March 2013.

[41] D. Adey, S. K. Agarwalla, C. M. Ankenbrandt et al., "Neutrinos from stored muons 2 STORM: expression of interest," http://arxiv.org/abs/1305.1419. 
[42] W. Winter, "Optimization of a very low energy neutrino factory for the disappearance into sterile neutrinos," Physical Review D, vol. 85, no. 11, Article ID 113005, 9 pages, 2012.

[43] P. Bernardini, A. Bertolin, C. Bozza et al., "Prospect for charge current neutrino interactions measurements at the CERN-PS," http://arxiv.org/abs/1111.2242.

[44] C. Rubbia, M. Antonello, D. Bagliani et al., "A comprehensive search for "anomalies" from neutrino and anti-neutrino oscillations at large mass differences $\left(\Delta m^{2} \sim 1 \mathrm{eV}^{2}\right)$ with two LArTPC imaging detectors at different distances from the CERNPS," SPSC-P-345, 2011.

[45] M. Antonello, D. Bagliani, B. Baibussinov et al., "Search for "anomalies" from neutrino and anti-neutrino oscillations at $\Delta m^{2} \sim 1 \mathrm{eV}^{2}$ with muon spectrometers and large LAr-TPC imaging detectors," http://arxiv.org/abs/1203.3432.

[46] CERN Neutrinos Study Group, ICARUS-NESSiE Collaborations, LAGUNA-LBNO Consortium, "Letter of Intent for the new CERN Neutrino Facility (CENF)," https://edms.cern.ch/ nav/P:CERN-0000077383:V0/P:CERN-0000096728:V0/TAB3.

[47] C. Rubbia, A. Guglielmi, F. Pietropaolo, and P. Sala, "Sterile neutrinos: the necessity for a 5 sigma definitive clarification," http://arxiv.org/abs/1304.2047.

[48] E. Balsamo, A. Bergnoli, A. Bertolin et al., "The OPERA RPCs front end electronics; a novel application of LVDS line receiver as low cost discriminator," Journal of Instrumentation, vol. 7, no. 11, Article ID P11007, 2012.

[49] R. Zimmermann, J. Ebert, C. Hagner et al., "The precision tracker of the OPERA detector," Nuclear Instruments and Methods in Physics Research A, vol. 555, no. 1-2, pp. 435-450, 2005, Erratum in Nuclear Instruments and Methods in Physics Research A, vol. 557, p. 690, 2006.

[50] T. Adam, E. Baussan, K. Borer et al., "The OPERA experiment target tracker," Nuclear Instruments and Methods in Physics Research A, vol. 577, no. 3, pp. 523-539, 2007.

[51] R. Acquafredda, T. Adam, N. Agafonova et al., "The OPERA experiment in the CERN to Gran Sasso neutrino beam," Journal of Instrumentation, vol. 4, no. 4, Article ID P04018, 2009.

[52] A. Bergnoli, E. Borsato, R. Brugnera et al., "Tests of OPERA RPC detectors," IEEE Transactions on Nuclear Science, vol. 52, no. 6, pp. 2963-2970, 2005.

[53] B. Pontecorvo, "Neutrino experiments and the question of leptonic-charge conservation," Soviet Physics-Journal of Experimental and Theoretical Physics, vol. 26, p. 984, 1968.

[54] Z. Maki, M. Nakagawa, and S. Sakata, "Remarks on the unified model of elementary particles," Progress of Theoretical Physics, vol. 28, no. 5, pp. 870-880, 1962.

[55] C. Andreopoulos, A. Bell, D. Bhattacharya et al., "The GENIE neutrino Monte Carlo generator," Nuclear Instruments and Methods in Physics Research A, vol. 614, no. 1, pp. 87-104, 2010.

[56] P. Huber, J. Kopp, M. Lindner, M. Rolinec, and W. Winter, "New features in the simulation of neutrino oscillation experiments with GLoBES 3.0. (General Long Baseline Experiment Simulator)," Computer Physics Communications, vol. 177, no. 5, pp. 432-438, 2007.

[57] N. Agafonova, A. Aleksandrov, O. Altinok et al., "Study of neutrino interactions with the electronic detectors of the OPERA experiment," New Journal of Physics, vol. 13, Article ID 053051, 2011. 

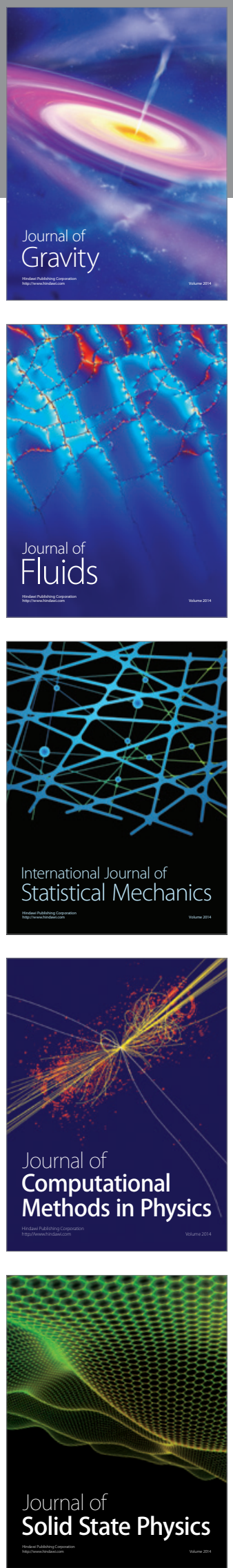

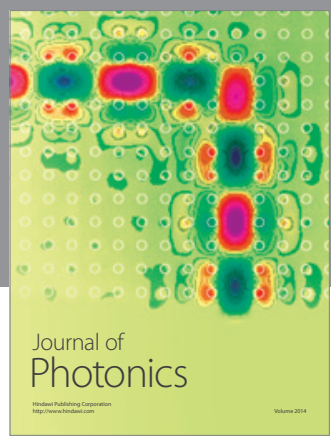

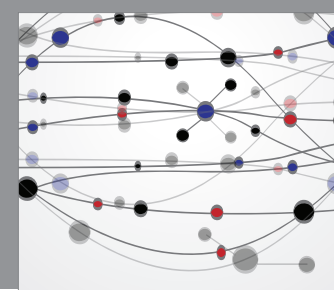

The Scientific World Journal

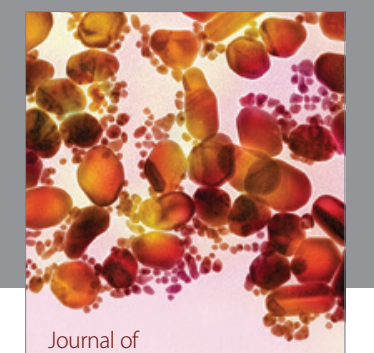

Soft Matter
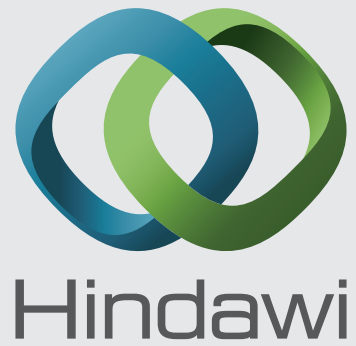

Submit your manuscripts at

http://www.hindawi.com
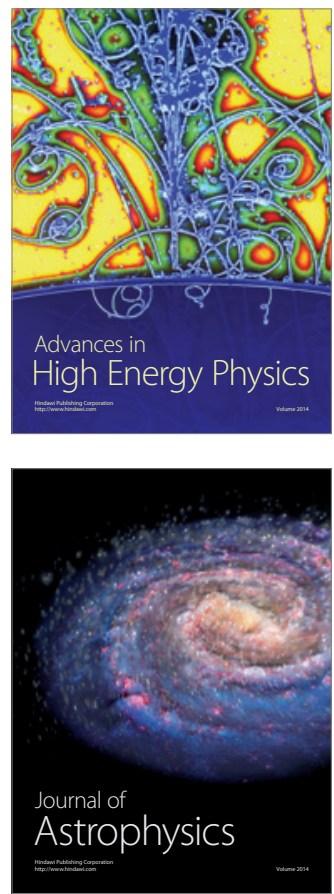
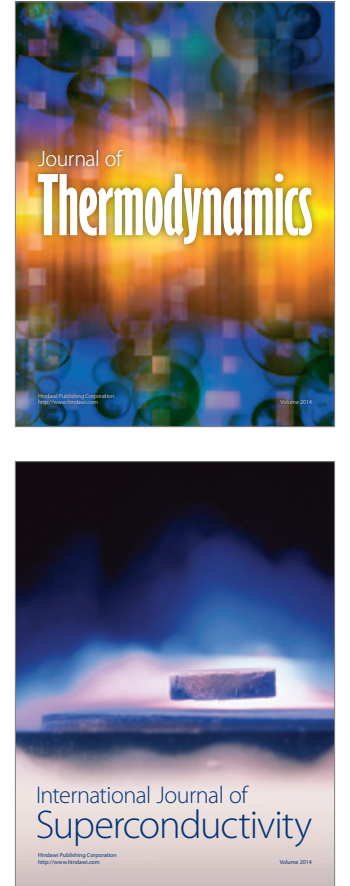
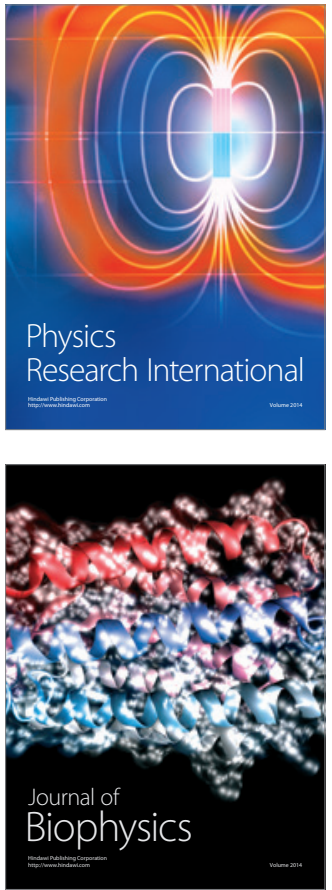
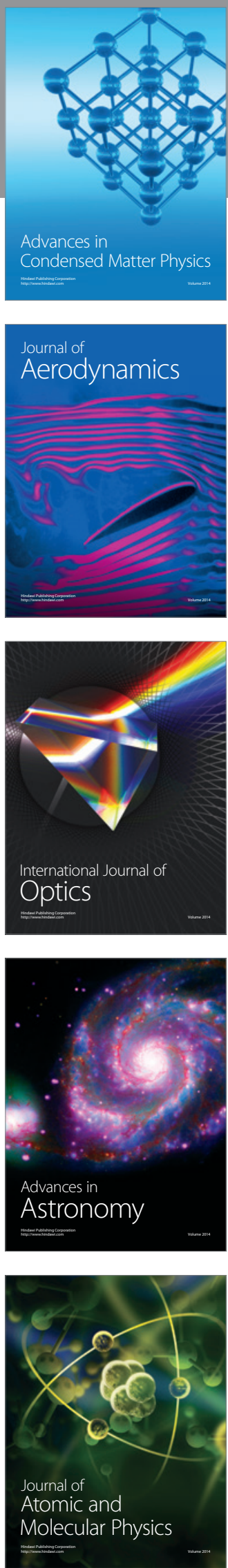